\title{
Toughness of natural rubber compounds under biaxial loading
}

Francesco Caimmi, Roberto Calabrò, Francesco Briatico-Vangosa, Claudia Marano, Marta Rink Chemistry, Materials and Chemical Engineering Department "Giulio Natta", Politecnico di Milano, P.za Leonardo da Vinci 32, I-20133 Milano, Italy

Engineering Fracture Mechanics 149 (2015) 250-261

\section{ABSTRACT}

Strain induced molecular orientation effect on the fracture toughness of natural rubber based compounds was studied under biaxial loading conditions, using non-linear elastic fracture mechanics. The J-integral at fracture was evaluated using the finite element method. Fracture toughness can be severely influenced by strain induced molecular orientation up to a material dependent threshold, above which toughness becomes constant.

The effect of the fracture phenomenology shown by two carbon black filled compounds, for which propagation is preceded by branching at the tip (sideways crack propagation), is shown to remove any significant stress concentration at the original crack tip, enhancing the apparent fracture resistance.

This is the post-print author version of the work.

Publisher's version of the paper is available at http://dx.doi.org/10.1016/j.engfracmech.2015.08.003

This work is licensed under Creative Commons Attribution Non-Commercial No Derivatives License 3.0 


\title{
Toughness of natural rubber compounds under biaxial loading
}

\author{
Francesco Caimmi ${ }^{\mathrm{a}, *}$, Roberto Calabrò ${ }^{\mathrm{a}}$, Francesco Briatico-Vangosa ${ }^{\mathrm{a}}$, Claudia Marano ${ }^{\mathrm{a}}$, Marta Rink $^{\mathrm{a}}$ \\ ${ }^{a}$ Chemistry, Materials and Chemical Engineering Department "Giulio Natta", Politecnico di Milano, P.za Leonardo da \\ Vinci 32, I-20133 Milano, Italy
}

\begin{abstract}
Strain induced molecular orientation effect on the fracture toughness of natural rubber based compounds was studied under biaxial loading conditions, using non-linear elastic fracture mechanics. The $J$-integral at fracture was evaluated using the finite element method. Fracture toughness can be severely influenced by strain induced molecular orientation up to a material dependent threshold, above which toughness becomes constant.

The effect of the fracture phenomenology shown by two carbon black filled compounds, for which propagation is preceded by branching at the tip (sideways crack propagation), is shown to remove any significant stress concentration at the original crack tip, enhancing the apparent fracture resistance.
\end{abstract}

Keywords: Natural Rubber Compounds, J-integral, biaxial tests

\section{Introduction}

It is well known that crystallising rubbers develop a strong strength anisotropy upon stretching: broadly speaking, if stretched along some direction they can become easier to break upon subsequent stretching perpendicularly to this direction [1-3]. This phenomenon is rather complicated and is linked with molecular orientation and stress induced crystallisation which have a significant impact on fracture toughness [4].

Gent and Kim [2] tested notched rubber strips that were stretched, before testing, in a direction perpendicular to the testing one; their grip system allowed them to keep the pre-stretching level fixed during testing. For crystallising rubbers, they found a very strong decrease of the tear energy of the material with increasing elongation.

In a previous work by some of the authors of this paper, natural rubber compounds were studied by applying a biaxial load using a cross-shaped specimen containing a central notch [3]. In those tests the samples were first stretched in a direction parallel to the notch, in order to induce a certain level of orientation in the material, and then the notch was opened by loading along the perpendicular direction. Different levels of orientation were induced in the material by applying different stretch levels during the first part of the test. The results were basically analysed in the framework of linear elastic fracture mechanics: an apparent critical stress intensity factor was evaluated from the fracture load at fracture initiation. The authors concluded that orientation produced a significant decrease in the resistance to crack propagation. Due to the non-linear nature of the materials used, such an analysis was to some extent not correct; in principle the trend observed could be apparent and caused by the use of a parameter which was not adequate to take into account the material behaviour and large deformations.

In addition to the uncertainties introduced by the analysis, further complications were brought in by the complex fracture phenomenology. Under certain conditions rubbers may exhibit a crack branching phenomenon that has been variously termed in literature as "sideways crack propagation" [3] or "knotty tearing" $[2,4,5]$. It takes place under load at the tip of a crack, where the front splits into two cracks that

\footnotetext{
* Corresponding author:

Email address: francesco.caimmi@polimi.it (Francesco Caimmi)
} 


\begin{tabular}{|ll|}
\hline \multicolumn{2}{|l|}{ Nomenclature } \\
$a_{0}$ & initial crack length \\
$B$ & specimens thickness \\
$C_{i}$ & constants for the description of branch shapes \\
$J$ & J-integral \\
$J_{c}$ & fracture toughness \\
$n$ & number of terms in Ogden's strain energy density function \\
$p$ & hydrostatic pressure \\
$P_{i}$ & components of first Piola-Kirchhoff stress tensor \\
$x, y$ & coordinate system \\
$U$ & strain energy \\
$W$ & specimen width \\
$\delta_{y}$ & boundary displacement \\
$\gamma_{i}$ & Ogden's model material parameters (stretch exponents) \\
$\lambda_{1}, \lambda_{2}, \lambda_{3}$ & principal stretches \\
$\lambda_{c}$ & stretch level needed to suppress sideways propagation \\
$\lambda_{x}, \lambda_{y}$ & stretches \\
$\mu_{i}$ & Ogden's model material parameter (modulus-like) \\
$\Psi$ & strain energy density \\
\hline
\end{tabular}

start to grow, more or less simultaneously, along an initial direction that is approximately perpendicular to the initial crack plane; often these sideways cracks follow a curved path for a short distance and then stop, due to the fact that, loosely speaking, the energy release rate associated with their propagation decreases while they become deeper (see [6] for an approximated analysis). After sideways crack propagation, if the load is further increased, propagation of a crack along the initial notch direction plane takes place; this subsequent fracture event will be termed "forward propagation", following the nomenclature proposed in [3]; often a very significant increment is needed.

Sideways cracks origin is not completely understood; it has been proposed that they may be linked with molecular orientation in front of the notch due to opening stresses [4], which therefore creates "weak planes" along the opening direction, but also that cavitation may play an important role in their formation because of the peculiar stress field that develops near the tip of a crack in hyperelastic materials [7]. On a similar line of reasoning, cavitation around carbon black particles may promote sideways crack formation [5]. Interestingly the phenomenon is suppressed if enough pre-stretch in a direction parallel to the initial notch is applied; the threshold level depends on the carbon black content [3]. Sideways cracks initiation and growth effectively reduces the stress concentration at the initial notch tip, thus greatly influencing fracture toughness.

This work builds upon the previous results by Marano et al. [3] by re-analysing them, this time in the framework of non-linear elastic fracture mechanics, hence using the critical $J$-integral, $J_{c}$, as a parameter to describe fracture resistance, as it has been widely done in literature (e.g. [6, 8-11]). The approach follows closely the linear elastic fracture mechanics one: fracture is assumed to take place when the $J$-integral reaches a critical value $J_{c}$. The $J$-integral can be evaluated using its definition, i.e. as the flux of the Eshelby's tensor, if the proper stress and deformation measures are used [12]. It is worth recalling that, at variance with the linear case, for flat and sharp crack problems involving non-linear material models and large deformations, there is no universal solution for the asymptotic crack tip fields as in the linear case, but the asymptotic behaviour depends on the constitutive law; a review of the available results can be found in the work by Gao et al. [13]. Generally speaking, as in the linear case, the displacement field still follows a power law as the distance from the crack tip tends to zero; the power law exponent however is not equal to $\frac{1}{2}$ but it depends on the material model; for instance for Ogden model [14], which is the one which will be used in this work, it depends on the largest stretch exponent in the strain energy function (see eq. 1)[15]. Usually, considering mode I plane problems, there is only one singular Cauchy stress component, the opening one, as, because of the finite deformations, the crack faces open deforming into some curve (the crack "blunts"). Therefore, owing to equilibrium, at the tip of the crack the only non-vanishing stress component is the one 
tangent to the crack faces ${ }^{1}$. On the other hand, the strain energy density is still singular and shows a $1 / r$ power-law behaviour, exactly as in the linear case, at least for all the hyperelastic models for which the stress grows indefinitely with deformation. That is, crack blunting due to the large deformation does not imply boundness of the stresses at the crack tip, and therefore the problem can be tackled, in principle, by assuming an infinitesimal process zone and a one parameter description of fracture. Some limits of this approach will be discussed in the rest of the paper.

In addition to the results on the cross shaped specimen, some tests were run using an ad-hoc developed square shaped specimen that can be fitted in a biaxial dynamometer. Both the tests are briefly reviewed in Sec.2.

In order to evaluate the $J$-integral in a non-linear setting, Ogden's model for isotropic isocoric hyperelastic materials [14] was chosen. The tests used to identify the material parameters are described in Section 2.2, while Section 2.5 describes the finite element (FE) models that were developed to calculate the $J$-integral in the square and cross shaped specimens. This was necessary as there are no available analytical solutions. The results of this analysis are presented in Section 3.1.

A brief analysis of the effect of sideways crack propagation on the elastostatic fields near the crack tip, again by using the FE method, is also presented in this work. Section 2.4 presents the methods used to approximately derive sideways crack shape in the undeformed configuration; this shape was used in a FE analysis to study their effect on $J$. The result are presented in Section 3.2.

\section{Materials and methods}

\subsection{Materials}

Natural rubber (NR0) and two carbon black filled natural rubber compounds (NR25 and NR50) were considered in this study. The filled compounds contain 25 and 50 phr of N330 carbon black. The materials were kindly supplied by Bridgestone T.C.E. (Roma, Italy). Sulphur content in the rubber was 1.3 phr, and in addition stearic acid (2 phr), tert-Butyl-2-benzothiazole (0.8 phr) and Zinc oxide (3 phr) were added to the rubbers for the vulcanisation process. Rubber sheets were compression moulded at $160{ }^{\circ} \mathrm{C}$ and $8 \mathrm{MPa}$ for $15 \mathrm{~min}$ so as to assure complete sulfur vulcanisation. Uniaxial tensile test-pieces were cut using a die from flat sheets, while biaxial and pure shear specimens (see section 2.2) were compression moulded in specially devised moulds. The nominal thickness of the sheets was $1 \mathrm{~mm}$.

\subsection{Material behaviour characterisation}

It was assumed that the natural rubber compounds used in this study could be described by Ogden's hyperelastic law for incompressibile materials [14]. The corresponding strain energy density is given by

$$
\Psi=\sum_{i=1}^{n} \frac{\mu_{i}}{\gamma_{i}}\left(\lambda_{1}^{\gamma_{i}}+\lambda_{2}^{\gamma_{i}}+\lambda_{3}^{\gamma_{i}}-3\right),
$$

where $\lambda_{j}(j=1,2,3)$ are the principal stretch ratios and $\mu_{i}$ and $\gamma_{i}$, with $i=1, \ldots, n$, are material parameters. The number of terms in the sum, $n$, may be regarded as a material parameter as well, however, it is common practice to fix it a priori; in this work $n$ was arbitrarily fixed to 3 . Hence a six parameter model was used.

The shear modulus in the undeformed configuration, $\mu$, satisfies the following relationship [14]

$$
2 \mu=\sum_{i=1}^{n} \mu_{i} \gamma_{i}
$$

The principal first Piola-Kirchhoff stresses are found from direct differentiation of Eq.1 as [16]:

\footnotetext{
${ }^{1}$ Stress measures referred to the undeformed configuration usually show three singular stress components as in the linear case, but generally different stress components present singularities with different strengths.
} 


$$
P_{i}=-\frac{p}{\lambda_{i}}+\frac{\partial U}{\partial \lambda_{i}},
$$

(no summation on $i$ ). In the last equation, $p$ is the hydrostatic pressure that can be determined by the stress boundary conditions pertaining to a specific load case [16].

In order to identify the model constants, three types of tests were employed: uniaxial, pure shear (PS) and equibiaxial tension.

Uniaxial tensile tests were run using a dumbbell test specimen as prescribed by ASTM D638 (type B-IV) and are described elsewhere [17].

PS tests (see e.g. [18]) were run on strips $100 \mathrm{~mm}$ wide with a $10 \mathrm{~mm}$ gauge length. These tests were run on an Instron 5800 dynamometer, at a prescribed crosshead speed of $30 \mathrm{~mm} \mathrm{~min}^{-1}$ (which provides a strain rate similar to that of the tensile tests in [17]).

Equibiaxial tensile tests were perfomed on unnotched square specimens $\left(2 a_{0}=0\right.$ in Fig.1(a)). The specimen edges are thicker than the rest, in order to connect the specimen to a biaxial dynamometer. Five separate mounting positions were prepared for each side of the specimen, corresponding to the five clamps on each side of the clamping system, by cutting the thick edge. At the end of each cut a circular hole was cut in the specimen to reduce the stress concentration. Similar solutions have been adopted in the past for biaxial tensile tests $[19,20]$. Nevertheless it is to be noted these cuts acts as stress raiser, reducing the range of strains for which the biaxial material behaviour can be measured, as they cause premature failure at the edges.

The tests were run on a custom-built dynamometer; the experimental setup can be seen in Fig. 1(b). The crosshead displacement rate was $60 \mathrm{~mm} \mathrm{~min}^{-1}$.

Further details on the biaxial dynamometer and on the characterisation tests can be found in [21]. Here only the uniaxial nominal stress-stretch curves are shown to give an idea of the different mechanical behaviour of the three compounds (Fig.2). As expected, the more carbon black is added, the stiffer the resulting material.

To identify the model parameters, data from uniaxial, pure-shear and equibiaxial tests were simultaneously fitted; the fitting procedure and its results are discussed in [21] where it is also shown, through a series of validation checks, that by using the identified material constants the mechanical behaviour of the materials is adequately described. The reader is therefore referred to that work for additional details and results. The identified parameters are given in Table 1.

As an example, the results of the fitting procedure are shown (solid lines) in Fig.2 for the uniaxial tensile test.

\subsection{Fracture Tests}

To study the effect of orientation on toughness, two fracture tests were considered in this work. As outlined in the Introduction, the fracture tests on cross shaped specimens performed by Marano et al. [3] were analysed anew by FEM; further, new fracture tests were performed on notched square specimens (Fig.1(a)). The reader is referred to the cited work for the details on the cross shaped specimen. Here it will suffice to recall that the dimensions are those given in Fig. 3. The dark areas in Fig. 3 are regions were a reinforced rubber was co-cured with the material to confer proper stiffness to the gripped region avoiding problems with material flow from the grips or slippage; they do not contribute significantly to deformation.

It should also be recalled that the tests were run in two steps: first a pre-stretch is applied by prescribing a fixed displacement rate to the arms parallel to the $x$ direction, up to some value of the displacement $\delta_{x}$ (Fig. 3) while keeping the load applied along $y$ as close as possible to zero; in the second step a fixed displacement rate is applied to the arms parallel to the $y$ direction while keeping the displacement of the transverse arms fixed. Before the second step the load in the $x$ direction was allowed to relax at a fixed displacement to an equilibrium value, although the visco-elastic behaviour of these rubber was not pronounced (and was not taken into account during modelling).

It is not easy to quantify into a single descriptor the molecular rubber orientation inside the material, especially considering the complex strain distribution near the crack tip. To keep the description of the 
phenomenon simple, in this work the nominal stretch ratio along the $x$ direction was chosen as an overall measure of the material orientation induced by the initial pre-stretch. It is given by

$$
\lambda_{x}=\frac{W+2 \delta_{x}}{W}
$$

where $W$ is shown in Fig.3. Henceforth such a stretch value will be simply referred to as "orientation".

Fracture tests were also run on notched square shaped samples (Fig.1(b)). The $16 \mathrm{~mm}$ long notch at the centre (along the $x$ direction) was inserted by a sharp razor blade mounted on a special fixture. The test procedure was the same as for the cross shaped specimen, and a definition of the nominal stretch ratio analogous to the one in Eq.4 was used, but with $W$ now given by the corresponding dimension in Fig.1(b) $(131 \mathrm{~mm})$. The crosshead displacement rate was set to $60 \mathrm{~mm} \mathrm{~min}^{-1}$.

Digital image correlation (DIC, see e.g. [22]) was used to measure the displacement field for square shaped fracture specimens. A random speckle pattern was drawn on the specimens with a water based metal grey paint, sprinkled by an airbrush; tests were video recorded at a frame rate of $1 \mathrm{fps}$ and with a resolution of $34 \mathrm{px} / \mathrm{mm}$. The step size chosen for the correlation analysis was about $0.15 \mathrm{~mm}$, with subset size $0.6 \mathrm{~mm}$. DIC was perfomed using the commercial software package VIC2D by Correlated Solutions, Inc.

In addition to the two fracture specimens described above, for comparison purposes fracture tests were also perfomed on PS specimens with an edge notch. These tests are thoroughly described in $[17,21]$; since here they are used only to estimate the shape and length of the sideways cracks and to compare the toughness values with the ones form the square and the cross tests, the reader is referred to the cited works for further details. As PS specimens do not contract (except for edge effects) along a direction parallel to the notch, they nominally corresponds to the case $\lambda_{x}=1$, using the orientation measure conventionally adopted in this work, i.e. to the case when the total length along the $x$ direction is held fixed.

All the test were perfomed at room temperature.

\subsection{Approximate determination of the sideways cracks shape}

Among the materials tested, NR25 and NR50 showed sideways crack propagation before propagation of the main crack, if the orientation was not enough to suppress it. In the deformed configuration the sideways crack appearance is shown in Fig.4(a). To insert sideways cracks in the finite element models (Sec.2.5), it was necessary to determine their shape. This was done by using DIC measurements. Next to the newly created free surfaces generated by sideways crack progression into the material, there is no more a random speckle pattern, so that a digital image correlation process becomes impossible. Such a correlation loss may be used to track the position of the sideways crack tip as a function of time with a precision mostly determined by DIC step size. It is thus possible to reconstruct the shape the sideways crack would have in the undeformed configuration. In a very schematic way the undeformed configuration they would assume if the samples were unloaded is shown in Fig.4(b): they tend to grow backwards (consistently with the numerical results in [23]) with respect to the initial notch direction. The same shape can also be observed post-mortem on the tested samples but it is harder to see as they tend to close upon unloading.

For crack branching in brittle materials, there are theoretical and experimental results on the shape branches should take. From Cotterell and Rice theoretical analysis [24] it is found that, for small kinks, the branch should be approximated by $y=C_{1} x\left(1+C_{2} x^{1 / 2}\right)$, where $C_{i}$ are some constants. Similar results were suggested by Adda-Bedia and co-workers (e.g. [25]), which found the following representation for the curve describing the crack path: $y=C_{3} x^{3 / 2}$. Sharon and Fineberg [26] measured the branches arising from dynamic propagation in a Polymethilmetacrilate and a Polycarbonate and found they both obeyed the following power law function: $y=0.2 x^{0.7}$, where lengths are in millimetres.

Although in brittle materials branches tend to partially follow the main crack path (they grow "forward") it was here assumed that a power law could be used as a very rough approximation to describe their shape in order to introduce them into a finite element model. In Fig.4(c) the measured sideways crack tip positions are shown (the reference system is given in Fig.4(b)). The plot shows data from both the PS fracture specimens [21] and the square shaped fracture specimens; the only material for which data could be measured was NR50, as for NR25 the sideways cracks were too small to be measured; in NR25 their maximum size was estimated to be less than $0.1 \mathrm{~mm}$. 
As expected for such a complex phenomenon, there is a lot of scatter in the sideways crack trajectories, and maybe the crack shapes for PS and square specimens are somewhat different. Nonetheless a power law fit of all the data was attempted resulting in the following power law: $|y|=C_{1}|x|^{C_{2}}$, where $C_{2}=0.53( \pm 0.09)$ and $C_{1}=1( \pm 0.15) \mathrm{mm}^{1-C_{2}}$. While the correlation coefficient is relatively small, being 0.43 , a more precise attempt at finding a more precise shape for the sideways cracks will not be pursued here, as this will have no significant influence on the main effect sideways cracks have (see Sec.3.2). Their shape will be assumed to follow the above given power law from now on. When needed, the sideways crack arc length, defined by

$$
s(x)=\int_{0}^{x} \sqrt{1+\left(C_{1} C_{2}|t|^{C_{2}-1}\right)^{2}} \mathrm{~d} t,
$$

will be used (see Fig.4(b)) to characterise their depth. The maximum value of $s$ found experimentally was about $0.8 \mathrm{~mm}$ in NR50 samples.

\subsection{Finite element simulations}

To calculate the $J$-integral FE models were developed using the commercial FE package ABAQUSC) [27]; they are shown in Fig.5.

For the cross shaped specimen a simple 2D non-linear elastic model was used exploiting specimen symmetry (Fig.5(a)). The reinforced region (dark areas in Fig.3) was modelled using a linear elastic material (whose modulus was measured in a tensile test) while for the central region Ogden's model was used. In a region of radius $5 \times 10^{-2} \mathrm{~mm}$ centred at the crack tip, elements with a characteristic length of about $5 \times 10^{-3} \mathrm{~mm}$ were used. Outside of such a region, concentric rings of elements with side length increasing in a logarithmic fashion were used, up to a radius of $1 \mathrm{~mm}$. A free meshing technique was used elsewhere. Eight-noded plane-stress rectangular elements were used (CPS8 in ABAQUS). A boundary displacement rate was prescribed at the arms ends, mimicking the experimental test. A convergence study was run checking convergence of the predicted load and of the $J$-integral, which was evaluated using ABAQUS built in routines [27]. The selected mesh comprised about 8200 elements.

As to the square shaped specimen, modelling the boundary conditions depicted in fig.1(b) would have been very complicated. To keep the model simple, only a rectangular slab with dimensions $60 \mathrm{~mm}$ x $22 \mathrm{~mm}$, whose mesh is shown in fig.5(b), was modelled. The slab is centred on the crack and near the right crack tip, where the $J$-integral was evaluated, meshing was the same as for the cross shaped sample case. A slightly coarser mesh was used for the left crack tip. The elements used were the same as in the cross shaped specimen model. As to the boundary conditions, the displacements measured by DIC were applied on the boundary of the FE model by exploiting standard submodelling techniques [27].

Using this approach, it is not possible to predict the boundary loads and to compare them with the experimental ones. In order to be able to make a comparison of some global quantities to get an estimate of the reliability of the simulations, the major and the minor diameters of the deformed crack were chosen [21]. A mesh convergence study was run by monitoring the convergence of these variables; the study lead to a model containing about 5800 elements. The same elements used for the other model were used.

For both the tests the simulations were run for a time corresponding to the fracture time, defined as the initiation of propagation of the main in crack in the direction parallel to the initial notch, as determined from the video-recordings.

For the evaluation of the influence of the applied orientation on $J_{c}$ sideways crack presence was disregarded and they were not introduced into the model.

Additional models with sideways cracks included were developed to study their effect on the elastostatic fields in the crack region. In order to stick to a purely non-linear elastic framework their growth was not modelled but they were introduced in the model from the beginning. Their shape was assumed to follow the expression given in Sec.2.4, which was replaced by a spline representation for drawing it in the FE program. Sideways cracks were modelled as flat and sharp cracks, i.e. as lines across which displacement discontinuities are allowed. The models were still assumed to be symmetric, thus the length $s$ of the two branches was assumed to be same. At the sideways crack tip, a mesh similar to the one used at the tip of the main crack was used. 


\section{Results and discussion}

\subsection{Effect of orientation on toughness}

The dependence of $J_{c}$ on the applied orientation level $\lambda_{x}$ is shown in Fig.6 for all the three materials. Cross and square symbols correspond to values evaluated from the cross and square shaped specimens respectively; triangles correspond to values measured with PS test [21], that are here assumed as reference values since an analytical solution is available for them.

For NR0 (Fig.6(a)), which did not show sideways crack propagation, $J_{c}$ decreases smoothly with orientation, from about $10 \mathrm{~N} \mathrm{~mm}^{-1}$ at $\lambda_{x}=1$ to about $1 \mathrm{~N} \mathrm{~mm}^{-1}$ for $\lambda_{x}=2.5$, which is a tenfold reduction. The values coming from different test geometries are well in line with each other over the whole range of applied $\lambda_{x}$, and the biaxial tests at $\lambda_{x}=1$ agree with the PS values. The trend is qualitatively similar to the one reported by Gent and Kim [2], although toughness values are different, and confirms the trends obtained by Marano et al. [3] using a linear elastic analysis.

For NR25 (Fig.6(b)), as said earlier, very small sideways cracks developed before propagation of the main crack. They could be no longer detected by inspection of the test video recordings for values of $\lambda_{x}>\lambda_{c}=1.3$ [3]. Although the toughness for $\lambda_{x}=1$ is smaller than for NR0, the trend looks qualitatively similar to the one obtained for the latter material. The values decrease smoothly (although there is some more scatter here) with increasing orientation, again to a value which is about $1 \mathrm{~N} \mathrm{~mm}^{-1}$ for large values of $\lambda_{x}$. There is also a rough agreement between the different test geometries considered. The trend is qualitatively in line with those found by Marano et al.

With respect to the initial crack dimensions, NR50 (Fig.6(c)) showed deep sideways cracks. Their formation was suppressed for $\lambda_{x}>\lambda_{c}=1.7$. Looking at the biaxial tests, the results are qualitatively similar to those of the other materials, with a very significant decrease from a value of about $20 \mathrm{~N} \mathrm{~mm}^{-1}$ for $\lambda_{x}=1$ to about $1 \mathrm{~N} \mathrm{~mm}^{-1}$ for large values of $\lambda_{x}$. The results from the biaxial tests are consistent but, for $\lambda_{x}=1$, they differ significantly from the reference PS value. Once again, the trends are qualitatively in line with those obtained in [3].

As to the effect of carbon black content, the addition of $25 \mathrm{phr}$ lowers the toughness for all the pre-stretch values, while NR50 shows improvement over NR0 for all $\lambda_{x}$, at least up to $\lambda_{c}$, i.e. as long as sideways cracks develop. Beyond that threshold, $J_{c}$ values become comparable for all the materials, scattered around $1 \mathrm{~N} \mathrm{~mm}^{-1}$.

The calculation of $J_{c}$ for these materials allowed confirming that strain induced anisotropy has a very strong effect on the material resistance to crack propagation. The very same phenomenon that has been conjectured to be source of sideways crack propagation [4], i.e. the development of weak planes due to the orientation and crystallisation of rubber molecules, may as well be cause of the strong toughness decrease with applied pre-stretch.

In contrast with the results obtained by Gent and Kim using a PS configuration with pre-stretching applied before the test [2], the present results seem to indicate that for the materials under study there is a limit value for toughness, independent of the carbon black content, while in the former case no evident plateau could be spotted, even if the experimental pre-stretch applied covered a range wider than in the present case. Whether or not this is due to differences in compounds, experimental setups or testing conditions is not clear.

While the overall findings by Marano et al. were confirmed by this new analysis, the question of discrepancies between the values measured by PS and by biaxial tests is still open. Quantitatively explaining it would be important in order to confirm the previous findings, which otherwise would still refer, at least as long as they regard results obtained after the development of sideways cracks, to a sort of apparent toughness, rather than to an actual material property (although "orientation" dependent).

As when sideways cracks are not there or are of negligible size there is no difference between different geometries, they are the principal suspects to be the discrepancy cause. In particular $s$ was similar for the tested geometries, but the ratio $a_{0} / s$ was rather different between them. The effect of sideways crack development and of their length on the calculation of the $J$-integral was investigated using the FEM method, and the results are presented in the next subsection. 


\subsection{Analysis of sideways cracks}

All the results that will be shown henceforth were calculated at the experimental displacement values corresponding to forward propagation, i.e. at the initiation of the main crack, after sideways propagation took place.

To study the effect of branches on initiation of a crack propagating along the original notch direction, the influence of sideways cracks on the elastostatic fields was analysed first. Fig.7(a) shows a detail of the deformed shape of the crack tip region of a cross shaped specimen with sideways cracks modelled at $\lambda_{x}=1$; only one quarter of the specimen is shown. $s$ was set to $0.78 \mathrm{~mm}$. The contour plot shows the strain energy density $\Psi$ (called SENER in ABAQUS) in MPa. There is a qualitative agreement between the deformed shape predicted and those observed experimentally (Fig.4(a)).

More insight can be gained by looking at the distribution of the strain energy density ahead of the crack tip, i.e. along $x$ at $y=0$ in a reference system centred at the original crack tip as in Fig.4(b), which is plotted in Fig.7(b). Results from the simulations of both PS and cross shaped specimens are shown.

When there are no sideways cracks, the strain energy density is singular and approaches the tip following a $1 / x$ power law, as indicated by the corresponding dashed line. This is exactly what is expected from known non-linear elastic solutions for most hyperelastic material models [13]. Note that linear elastic materials show the same strain energy density singularity. If sideways cracks are added to the PS model, it becomes harder to tell if the strain energy density is still singular; however, if it were singular, it would show a weaker singularity (about $1 / x^{0.1}$ as indicated by the dashed lines). Similar results were obtained for the cross shaped specimen, although the singularity seems to be slightly stronger. This result is similar to what one would expect by analogy with the linear elastic wedge case; while it was somewhat expected, such a result is definitely not obvious, as the only theoretical results on the asymptotic behaviour of the elastostatic fields at wedges and notches in hyperelastic materials are for other models of the strain energy function [13, 28]. The result would hold whatever is the actual shape of the sideways cracks, as long as they are assumed to initiate at the original crack tip.

As the strain energy, i.e. the product of the stress components and of the displacement derivatives, is less singular than $1 / x$, it immediately follows that at the tip of the original crack the $J$-integral must vanish (e.g. [29]), exactly as it happens in the linear elastic wedge case ${ }^{2}$. In this sense the development of the sideways cracks completely shields the crack tip, strongly reducing the stress intensity near the original crack tip and providing a huge improvement in the apparent material resistance to crack propagation. However, since the $J$-integral vanishes, it can no longer be used to study the onset of the main crack after sideways crack propagation has taken place, at least without having to resort to some $a d$-hoc additional criterion, as for example the maximum opening stress criterion or the so called "stress at a distance criteria". Such a criterion was used for instance by Williams [31] to study fracture under moderately large deformation for very soft materials - with a ratio of the cohesive stress $\sigma_{c}$ over the elastic modulus in the undeformed configuration $E$ which is less than one - but cannot be immediately extended to the present case: deformation is not small and even using a lower bound estimate of the cohesive stress, $\sigma_{c}$, by taking the (Cauchy) failure stress in uniaxial tension, $\sigma_{c} / E$ would be many times larger than one, because of strain induced crystallisation. For NR50, for example, $\sigma_{c} / E$ is over thirty; see Fig.2 and Tab.1, and recall that for uniaxial tension Cauchy stress is in this case given by $\sigma_{y}=P_{y} \lambda_{y}$.

Moreover, the (small) differences in the singularity strength cannot help explaining the different values of the measured fracture toughness and therefore in this case it is possible only to speak of an "apparent" value of $J_{c}$, an elastic analysis being inadequate in this case.

To understand the different NR50 $J$ values for PS and biaxial specimens measured at forward initiation, it was checked if the value of $J$ on a large contour completely enclosing the sideways cracks was the same irrespective of test configuration; this is equivalent to assuming that sideways cracks develop satisfying some kind of autonomy hypothesis, i.e. they always grow more or less in the same way provided a far field value of

\footnotetext{
${ }^{2}$ The vanishing of the $J$-integral was also verified by the compliance method [30], i.e. by extending the main crack tip along the $x$ direction at fixed boundary displacement and studying the variation of the total strain energy $U$ as a function of the crack length $a$. The study confirmed that the total strain energy has a stationary point for $a=a_{0}$ when sideways cracks are introduced, i.e. that $J \propto-\partial U / \partial a=0$.
} 
$J$ reaches some critical value. The idea comes from the fact that when sideways cracks are very small, as for NR25, they seem to have no influence on the apparent toughness, and from the fact that the values of $s$ at fracture initiation were similar for the different geometries. It was therefore checked if, at forward fracture initiation, a geometry independent value of the $J$-integral could be obtained on a large contour enclosing both the original and the sideways crack tips $\left(\Gamma_{1} \text { in Fig. } 7(\mathrm{c})\right)^{3}$. The results are shown in Fig.7(d) as a function of $s$, for two different tests. For the PS specimen the result is almost insensitive to the presence or length of the sideways cracks, while for the cross shaped specimen there is some moderate effect: $J$ increases as the sideways cracks become deeper, but it quickly reaches a constant value well before even getting close to the PS value ${ }^{4}$.

Therefore not even the use of an apparent $J$-integral can explain the differences in the values calculated for different NR50 samples. For deep sideways cracks therefore the actual material toughness cannot be properly evaluated while sticking to the framework of non-linear elastic fracture mechanics; while for the NR25 compound considered in this work this seems not to be an issue, the values obtained for the NR50 compound when $\lambda_{x}<\lambda_{c}$ are not a significant material parameter, as probably are all the values obtained when sideways crack propagation is involved. Further analysis, using alternative criteria [31] or using a cohesive zone approach (e.g. as done by Hui et al.[32]) are left as refinements for future work, since they would need a different approach to quantitatively explain the differences between toughness values recorded using different geometries on NR50.

\section{Summary and concluding remarks}

In this work previous results relevant to fracture tests under biaxial loading conditions were analysed anew in the framework of non-linear elastic fracture mechanics, and paired with some new results on a different test set-up, to study how the strain induced strength anisotropy influences toughness.

The results confirmed the findings of the previous work by Marano et al., and are in line with other literature results: fracture toughness, here interpreted in the framework of non-linear fracture mechanics as $J_{c}$, is severely affected by orientation, leading to a ten fold reduction for unfilled natural rubber when moving from a material which is not oriented to the maximum orientation studied. For natural rubber filled with $50 \mathrm{phr}$ of carbon black the effect is even stronger.

In this latter case however only an "apparent" toughness could be measured for a low level of orientation, as, because of the peculiar fracture phenomenology (sideways crack propagation). As shown by the FEM analysis the $J$-integral vanishes at the tip of a crack which exhibits sideways propagation and therefore the results can no longer be analysed in the framework of non-linear fracture mechanics. A possible way out of such an impasse would be the use of a Dudgale-like cohesive zone ahead of the crack tip, in order to reintroduce an energy sink in the model.

For filled compounds, when the orientation exceeds some threshold, which depends on the carbon black content and above which sideways crack propagation becomes suppressed, $J_{c}$ quickly falls to a value which is about $1 \mathrm{~N} \mathrm{~mm}^{-1}$, irrespective of the carbon black content. The same $J_{c}$ value is also reached by unfilled natural rubber for similar orientation levels.

\section{References}

[1] J. H. Fielding, Flex life and crystallization of synthetic rubber, Industrial \& Engineering Chemistry 35 (12) (1943) 1259 1261. arXiv:http://pubs.acs.org/doi/pdf/10.1021/ie50408a008, doi:10.1021/ie50408a008. URL http://pubs.acs.org/doi/abs/10.1021/ie50408a008

[2] A. Gent, H. Kim, Tear strength of stretched rubber, Rubber Chemistry and Technology 51 (1) (1978) 35-44.

\footnotetext{
${ }^{3}$ The calculations were made using an ABAQUS post-processing routine written in-house.

${ }^{4}$ It may be interesting to note that the main contribution to the far field $J$ comes from the integral along the crack lips (on $\left.\Gamma_{5,7}\right)$ where there is a strong strain energy concentration (see Fig.7(a)). It was also verified that the integral along $\Gamma_{6}$ tends to zero with the radius of the contour, in line with the previously mentioned results.
} 
[3] C. Marano, R. Calabrò, M. Rink, Effect of molecular orientation on the fracture behavior of carbon black-filled natural rubber compounds, Journal of Polymer Science Part B: Polymer Physics 48 (13) (2010) 1509-1515.

URL http://onlinelibrary.wiley.com/doi/10.1002/polb.22054/full

[4] G. R. Hamed, Molecular aspects of the fatigue and fracture of rubber, Rubber Chemistry and Technology 67 (3) (1994) 529-536. doi:10.5254/1.3538689.

URL http://dx.doi.org/10.5254/1.3538689

[5] A. Gent, C. Pulford, Micromechanics of fracture in elastomers, Journal of Materials Science 19 (11) (1984) 3612-3619. doi: $10.1007 /$ BF02396933. URL http://dx.doi.org/10.1007/BF02396933

[6] A. N. Gent, M. Razzaghi-Kashani, G. R. Hamed, Why do cracks turn sideways?, Rubber Chemistry and Technology 76 (1) (2003) 122-131. doi:10.5254/1.3547727. URL http://dx.doi.org/10.5254/1.3547727

[7] C. J. Quigley, D. M. Parks, The finite deformation field surrounding a mode i plane strain crack in a hyperelastic incompressible material under small-scale nonlinearity, International Journal of Fracture 65 (1994) 75-96. doi:10.1007/ BF00017144. URL http://dx.doi.org/10.1007/BF00017144

[8] K. Tsunoda, J. Busfield, C. Davies, A. Thomas, Effect of materials variables on the tear behaviour of a non-crystallising elastomer, Journal of Materials Science 35 (20) (2000) 5187-5198. doi:10.1023/A:1004860522186. URL http://dx.doi.org/10.1023/A\%3A1004860522186

[9] C. Netzker, T. Horst, K. Reincke, R. Behnke, M. Kaliske, G. Heinrich, W. Grellmann, Analysis of stable crack propagation in filled rubber based on a global energy balance, International Journal of Fracture 181 (1) (2013) 13-23. doi:10.1007/ s10704-013-9816-5. URL http://dx.doi.org/10.1007/s10704-013-9816-5

[10] M. Boggio, C. Marano, M. Rink, Time dependence of fracture behaviour of carbon black filled natural rubber, in: N. Jerrams, S.and Murphy (Ed.), Constitutive Models for Rubber VII, CRC Press, London, 2011, pp. 363-368.

[11] F. Caimmi, R. Calabrò, C. Marano, M. Rink, Biaxial fracture testing of rubber compounds, in: S. Jerrams, N. Murphy (Eds.), Constitutive Models for Rubber VII, CRC Press, London, 2011, pp. 449-454.

[12] J. D. Eshelby, Energy relations and the energy momentum tensor in continuum mechanics, in: M. Kanninen, W. Adler, A. R. Rosenfield, R. I. Jaffee (Eds.), Inelastic behaviour of solids, McGraw-Hill, 1970, Ch. 2, pp. 77-114.

[13] Y.-C. Gao, M. Jin, G.-S. Dui, Stresses, singularities, and a complementary energy principle for large strain elasticity, Applied Mechanics Reviews 61 (3) (2008) 030801. doi:10.1115/1.2909715. URL http://link.aip.org/link/?AMR/61/030801/1

[14] R. W. Ogden, Large deformation isotropic elasticity - on the correlation of theory and experiment for incompressible rubberlike solids, Proceedings of the Royal Society of London. Series A, Mathematical and Physical Sciences 326 (1567) (1972) pp. 565-584.

URL http://www.jstor.org/stable/77930

[15] K. Le, H. Stumpf, The singular elastostatic field due to a crack in rubberlike materials, Journal of Elasticity 32 (1993) 183-222. doi:10.1007/BF00131660 URL http://dx.doi.org/10.1007/BF00131660

[16] G. Holzapfel, Nonlinear Solid Mechanics, Wiley, Chichester, UK, 2000.

[17] C. Marano, M. Boggio, E. Cazzoni, M. Rink, Fracture phenomenology and toughness of filled natural rubber compounds via the pure shear test specimen, Rubber Chemistry and Technology 87 (3) (2014) 501-515. doi:10.5254/rct.14.86950. URL http://dx.doi.org/10.5254/rct.14.86950

[18] D. Charlton, J. Yang, K. Teh, A review of methods to characterize rubber elastic behavior for use in finite element analysis, Rubber chemistry and technology 67 (3) (1994) 481-503.

[19] C. W. Extrand, A. N. Gent, Strenght under various modes of deformation, International Journal of Fracture 48 (1991) $281-297$.

[20] K. Miller, Testing elastomers for hyperelastic material models in finite element analysis, Tech. rep., Axel Products, Inc.

[21] R. Calabró, Mechanical characterization of elastomers under quasi-static and dynamic biaxial loading conditions, Ph.D. thesis, Politecnico di Milano, Milano (2013).

[22] M. Sutton, W. Wolters, W. Peters, W. Ranson, S. McNeill, Determination of displacements using an improved digital correlation method, Image and Vision Computing 1 (3) (1983) 133-139. doi: http://dx.doi.org/10.1016/0262-8856(83) 90064-1.

URL http://www.sciencedirect.com/science/article/pii/0262885683900641

[23] R. Denzler, Computational configurational mechanics, Ph.D. thesis, Technischen Universität Kaiserslautern, Kaiserslautern (2006)

[24] B. Cotterell, J. Rice, Slightly curved or kinked cracks, International Journal of Fracture 16 (2) (1980) $155-169$. URL http://dx.doi.org/10.1007/BF00012619

[25] E. Katzav, M. Adda-Bedia, R. Arias, Theory of dynamic crack branching in brittle materials, International Journal of Fracture 143 (2007) 245-271. doi:10.1007/s10704-007-9061-x. URL http://dx.doi.org/10.1007/s10704-007-9061-x

[26] E. Sharon, J. Fineberg, Confirming the continuum theory of dynamic brittle fracture for fast cracks, Nature 397 (6717) (1999) 333-335.

URL http://dx.doi.org/10.1038/16891

[27] Simulia Corporation, Abaqus Analysis User's Manual. Version 6.11, Simulia Corp., 2011. 
[28] M. Arfaoui, K. Mansouri, A. Rezgui, An asymptotic finite plane deformation analysis of the elastostatic fields at a notch vertex of an incompressible hyperelastic material, Comptes Rendus Mécanique 336 (9) (2008) 737 - 743. doi:http: //dx.doi.org/10.1016/j.crme.2008.07.003.

URL http://www.sciencedirect.com/science/article/pii/S1631072108001174

[29] J. Hutchinson, On steady quasi-static crack growth, Tech. Rep. AFSOR-TR74-1042, Air Force Office of Scientific Research (1974).

[30] A. Corigliano, Damage and fracture mechanics techniques for composite structures, in: I. M. R. Karihaloo (Ed.), Comprehensive Structural Integrity, Vol. 3, Pergamon, Oxford, 2003, Ch. 9, pp. 459 - 539. doi:http://dx.doi.org/10.1016/ B0-08-043749-4/03041-X.

URL http://www.sciencedirect.com/science/article/pii/B008043749403041X

[31] J. G. Williams, Stress at a distance fracture criteria and crack self-blunting in rubber, International Journal of Non-Linear Mechanics 68 (2014) 33-36. doi:http://dx.doi.org/10.1016/j.ijnonlinmec.2014.06.008.

URL http://www.sciencedirect.com/science/article/pii/S0020746214001280

[32] C.-Y. Hui, J. A., S. J. Bennison, J. D. Londono, Crack blunting and the strength of soft elastic solids, Proceedings of the Royal Society of London A: Mathematical, Physical and Engineering Sciences 459 (2034) (2003) 1489-1516. doi: $10.1098 / \mathrm{rspa} .2002 .1057$. 


\section{List of Figures}

Biaxial tensile test . . . . . . . . . . . . . . . . . . . . . . . . 13

2 Uniaxial mechanical beahviour of the three compounds. Experimental data (symbols) and

Ogden's model fit (solid lines). . . . . . . . . . . . . . . . . . . . . . 13

3 Schematics of the cross shaped specimen. Dimensions in mm . . . . . . . . . . . . . . . 14

4 Sideways crack shape . . . . . . . . . . . . . . . . . . . . . . . . . . 15

$5 \quad$ FE models of fracture specimens. Dimensions in $\mathrm{mm} \ldots \ldots \ldots \ldots$

6 Fracture toughness vs. orientation along the notch direction. . . . . . . . . . . . 17

7 Sideways cracks: FEM results. . . . . . . . . . . . . . . . . . . . . . . 18 


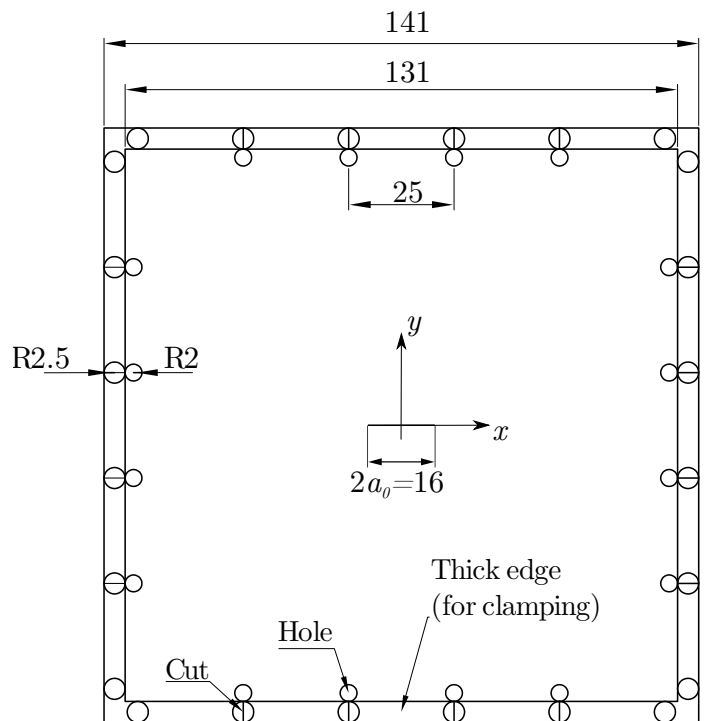

(a) Square shaped specimen used for biaxial tests: nominal dimensions in $\mathrm{mm}$.

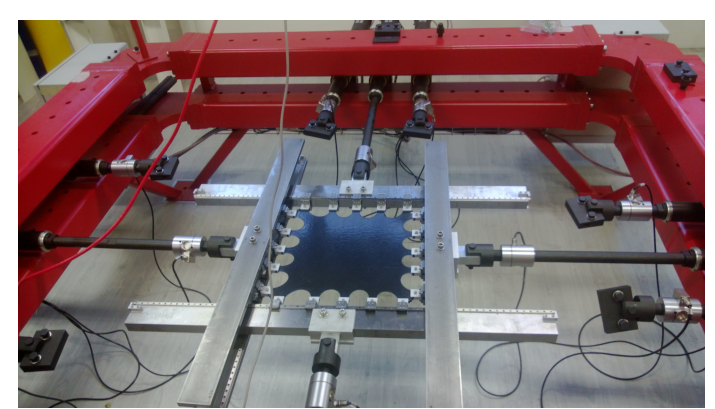

(b) The biaxial dynamometer with the loading rig used to test square shaped specimens.

Figure 1: Biaxial tensile test

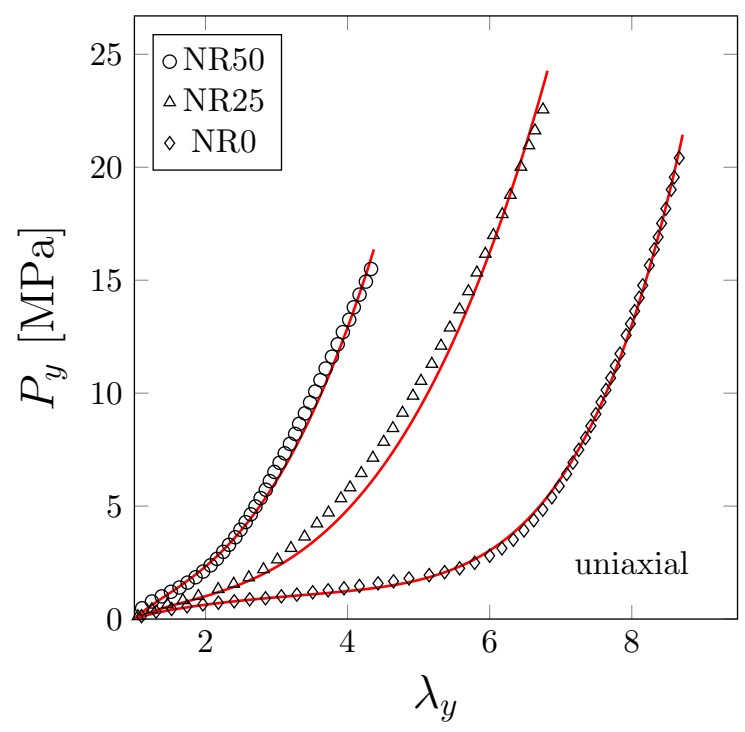

Figure 2: Uniaxial mechanical beahviour of the three compounds. Experimental data (symbols) and Ogden's model fit (solid lines). 


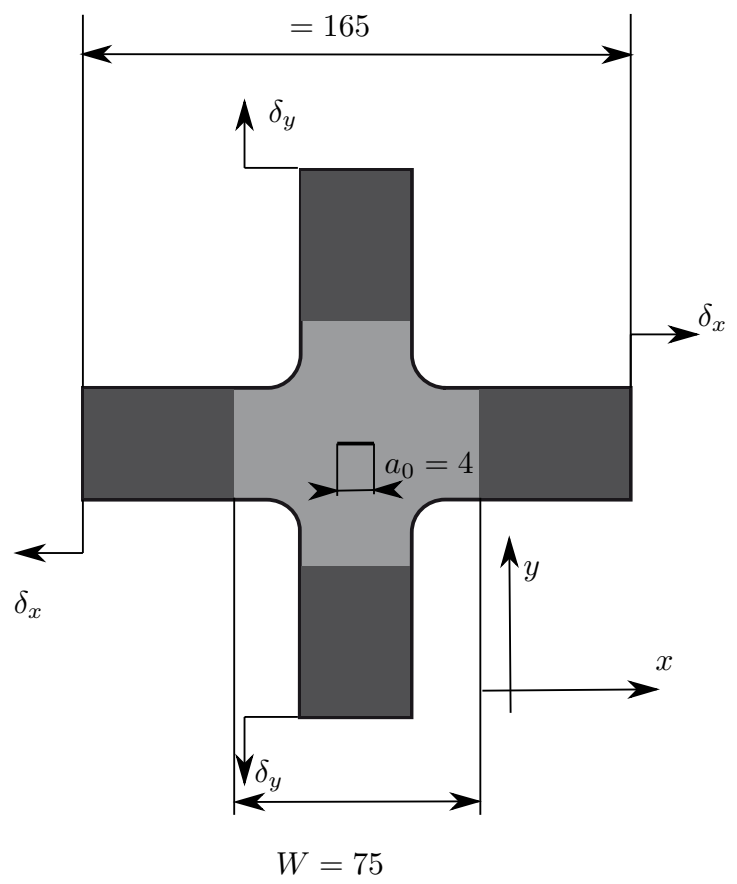

Figure 3: Schematics of the cross shaped specimen. Dimensions in $\mathrm{mm}$ 

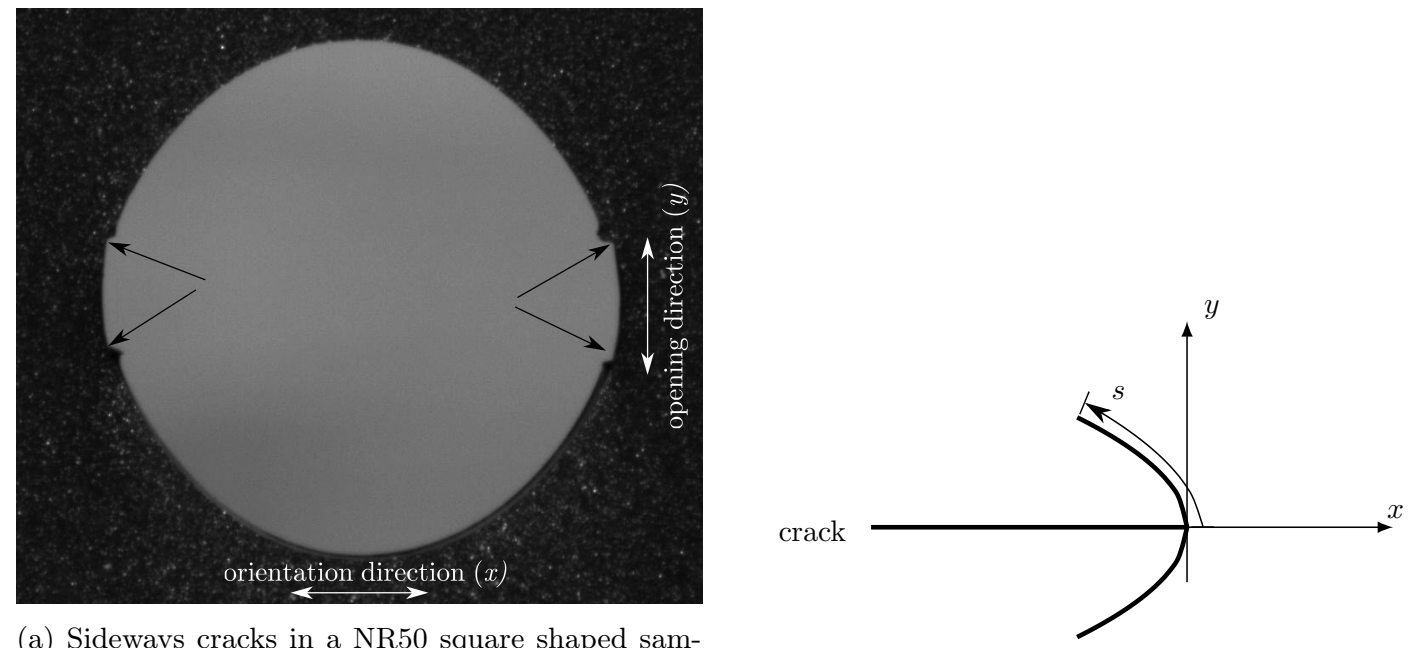

(a) Sideways cracks in a NR50 square shaped sample, just before onset of the main crack. The arrows indicate the sideways crack tip positions. $\lambda_{x}=1.2$.

(b) Schematic representation of sideways cracks in the undeformed configuration.

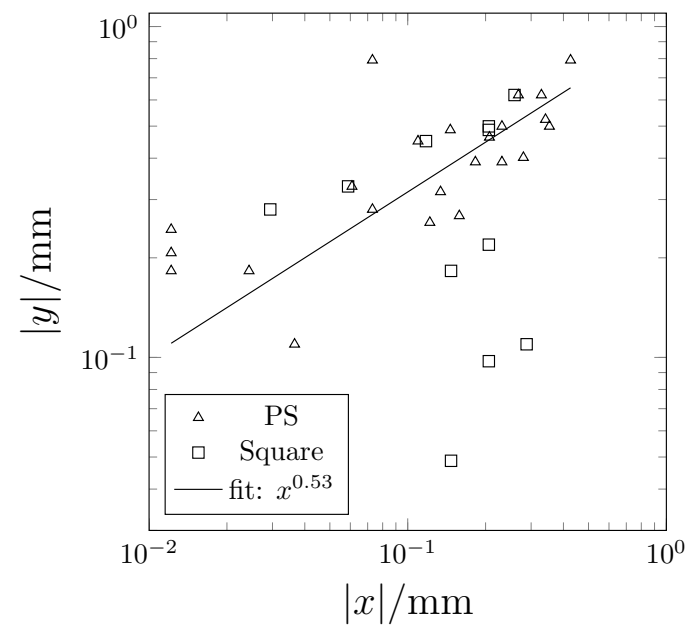

(c) NR50. Measured positions of the sideways crack fronts (symbols) and power law fit of the data (continuous line). Values from different specimens and at different times.

Figure 4: Sideways crack shape 


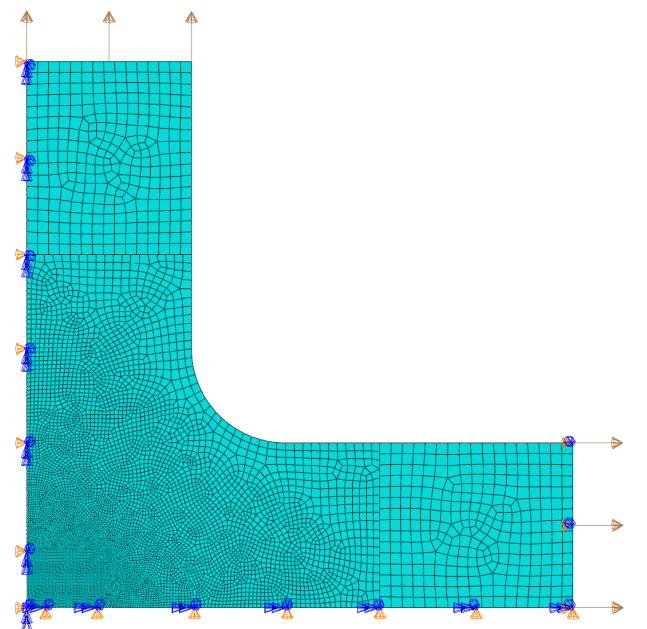

(a) Cross shaped specimen FE model. Only a quarter of the specimen was modelled.

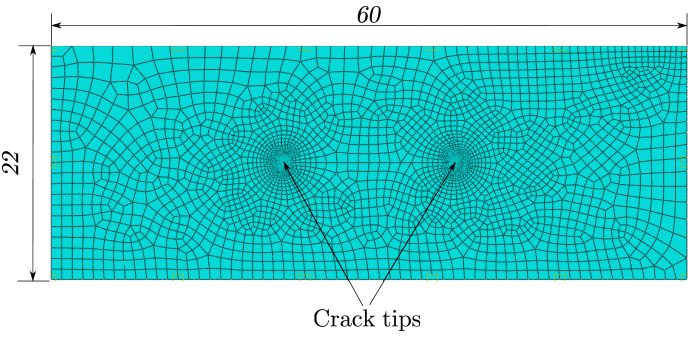

(b) Biaxial fracture specimen: submodel.

Figure 5: FE models of fracture specimens. Dimensions in $\mathrm{mm}$ 


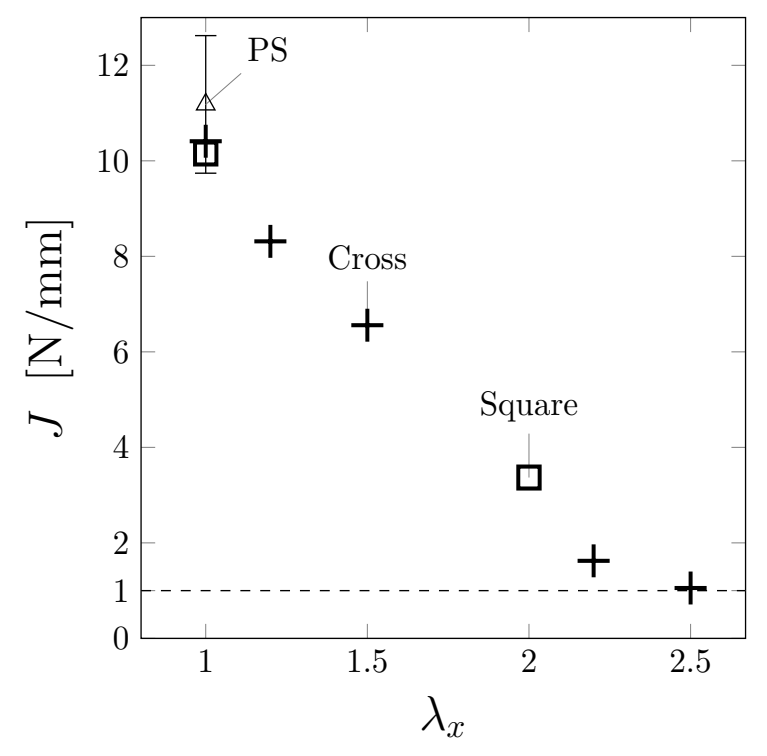

(a) NR0

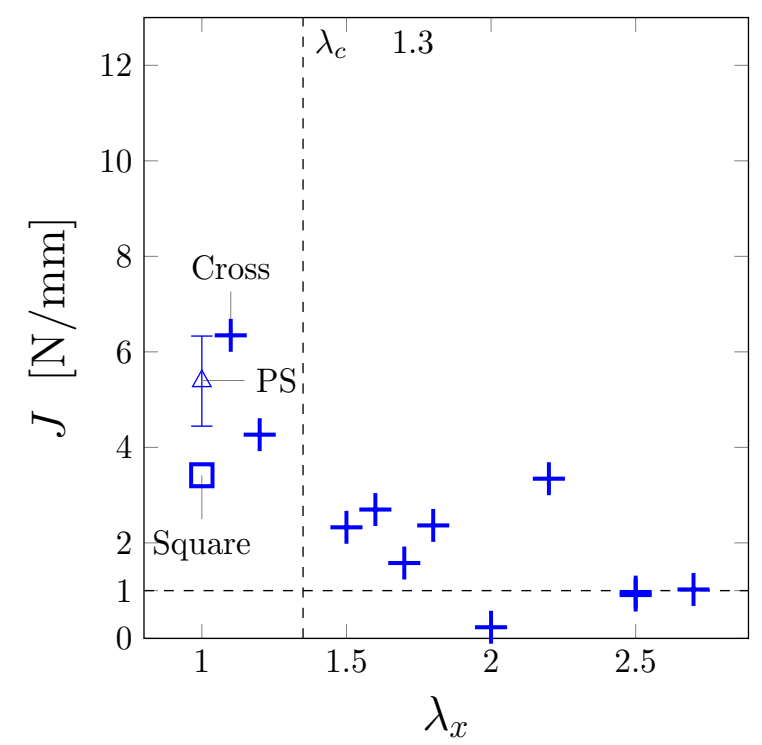

(b) NR25

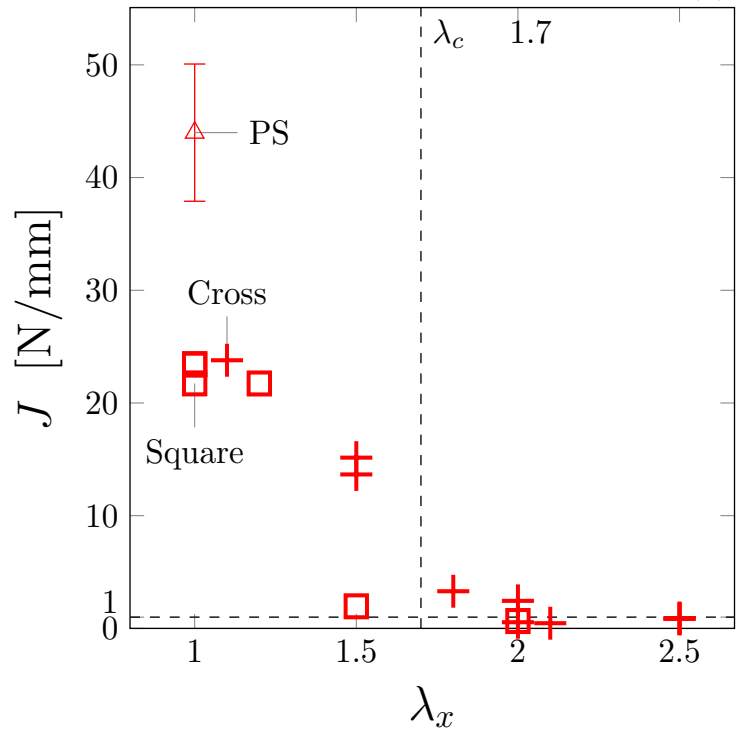

(c) NR50

Figure 6: Fracture toughness vs. orientation along the notch direction. 


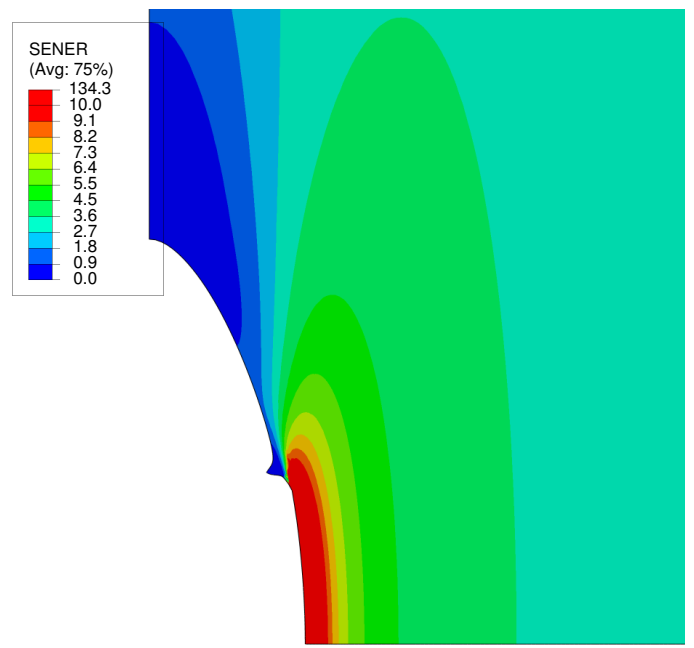

(a) Detail of the deformed configuration of the crack tip region with sideways cracks modelled $(s=0.78$ $\mathrm{mm})$. Contour of the strain energy density $\Psi$ in $\mathrm{MPa}$ (SENER in ABAQUS). NR50, cross shaped specimen.

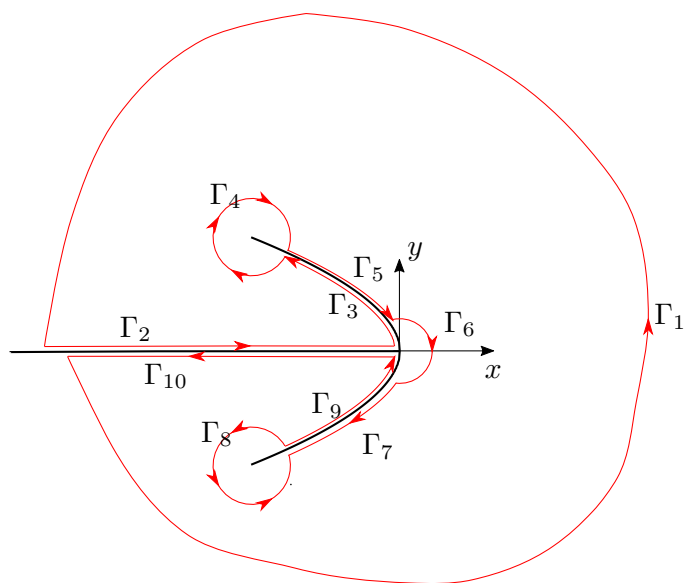

(c) Contours around the branched crack tip.

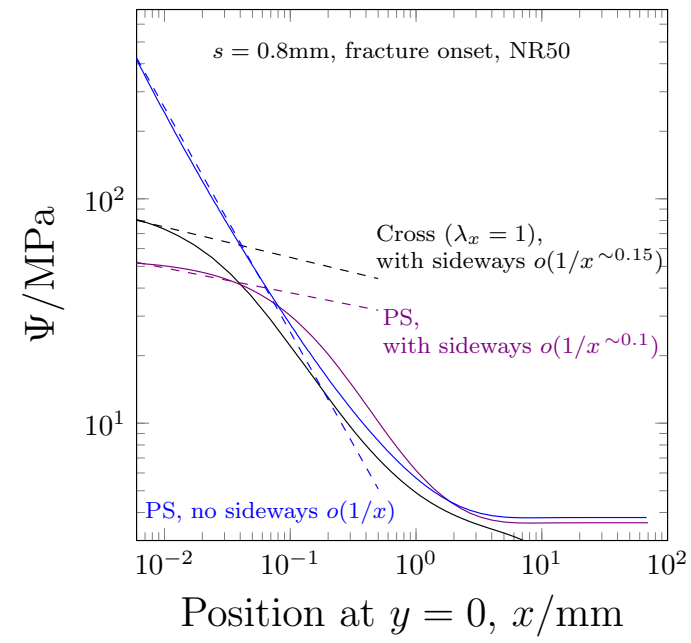

(b) Strain energy distribution on the crack plane ahead of the crack tip. Continuous lines: FE results. Dashed lines: power law fits.

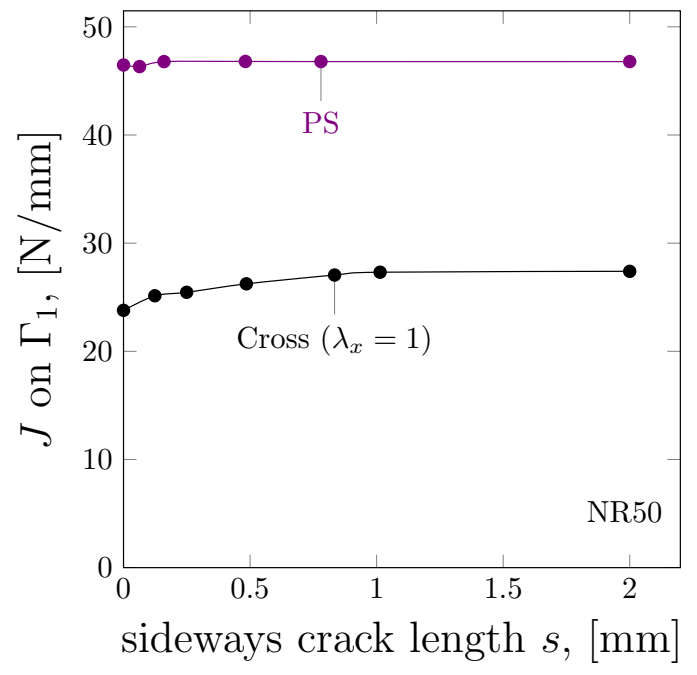

(d) $J$ on $\Gamma_{1}$ as a function of the sideways crack length.

Figure 7: Sideways cracks: FEM results. 


\section{List of Tables}

1 Ogden's model identified parameters for the three compounds. . . . . . . . . . . . . . . . . 20 


\begin{tabular}{l|cccccc}
\hline Compound & $\mu_{1}[\mathrm{MPa}]$ & $\mu_{2}[\mathrm{MPa}]$ & $\mu_{3}[\mathrm{MPa}]$ & $\gamma_{1}$ & $\gamma_{2}$ & $\gamma_{3}$ \\
\hline NR0 & 0.39 & 0.036 & -0.038 & 1.96 & 5.80 & 5.77 \\
NR25 & 0.94 & 0.041 & $-3.5 \mathrm{e}-5$ & 1.00 & 4.31 & -5.65 \\
NR50 & 1.39 & 0.29 & $-1.4 \mathrm{e}-4$ & 0.78 & 3.84 & -6.96 \\
\hline
\end{tabular}

Table 1: Ogden's model identified parameters for the three compounds. 